\title{
Synthesis and Physical Characterization of PMMA/PP and PMMA/ PAN Composites for Denture Applications
}

\author{
${ }^{1}$ Hassan A. Sharhan*, ${ }^{1}$ Zaynab N. Rasheed, ${ }^{2}$ Jawad K. Oleiwi \\ ${ }^{1}$ Department of Applied Sciences, University of Technology, Iraq \\ ${ }^{2}$ Materials Engineering Department, University of Technology, Iraq
}

\begin{tabular}{l}
\hline Article information \\
\hline Article history: \\
Received: June, 03, 2021 \\
Accepted: August, 04, 2021 \\
Available online: September, 18, 2021 \\
\hline
\end{tabular}

\section{Keywords:}

Poly (Methyl methacrylate),

Composite Materials,

Polypropylene (PP) fibers,

Poly acrylonitrile fibers (PAN)

*Corresponding Author:

Hassan A.Sharhan*

as.18.36@grad.uotechnology.edu.iq

\begin{abstract}
This study involves on manufacture of denture base resin with advanced physical properties through the addition of two type of synthetic fibers used as reinforcing materials polypropylene (PP) and Poly acrylonitrile (PAN). In this research, groups of three samples of both PMMA/PP and PMMA/PAN composites were prepared with the selected fiber weight ratios of $1.5,3.5,5.5$ and $7.5 \% \mathrm{Wt}$. Physical properties such as water absorption, thermal conductivity and density were evaluated under normal conditions. The chemical bond structure was also investigated for all samples using FTIR test. The results showed that when fibers ratio increased until $7.5 \% \mathrm{Wt}$., the water absorption increases, as well for each fiber which was over $0.6 \%$ compared to the $0.3 \%$ of the neat sample. A noticeable decrease in the thermal conductivity property from 0.33 $\mathrm{W} / \mathrm{m} . \mathrm{K}$ for PMMA/PP and $0.24 \mathrm{~W} / \mathrm{m}$.K for PMMA/PAN comparing to $0.46 \mathrm{~W} / \mathrm{m}$. K of the neat polymer was revealed. As for the density results, it was found that when the fibers ratios were increased, the sample density slightly decreased and reached $1.09 \mathrm{~g} / \mathrm{cm}^{3}$ for PMMA/PP and $1.1 \mathrm{~g} / \mathrm{cm}^{3}$ for PMMA/PAN at the maximum fibers rate. FTIR results indicated there was no new peaks appeared after reinforcement with both fibers. This could refer to the good physical bond between the mixtures, no new material formed.
\end{abstract}

DOI: 10.53293/jasn.2021.3759.1040, Department of Applied Science, University of Technology

This is an open access article under the CC BY 4.0 License

\section{Introduction}

The study of the chemical composition and properties of designer materials used in dentistry and the surrounding environment is known as dental materials science [1]. Acrylic resins are the most common material used in the manufacture of dentures since the year 1937. It has properties that make it the best among other resins such as low economic cost, ease of formation at room temperature [2], hypoallergenic, biocompatible, non-toxic, non-irritating, mechanically stable, corrosion resistant, non-volatile over time, dimensionally stable, and little difference with solvent and temperature [3]. However, because to certain physical features such as polymerization shrinkage and water sorption, and to overcome the degraded physical qualities of PMMA, more advancements are required [4]. The resin was thus strengthened through the addition of some fillers to the PMMA matrix materials thus forming an enhanced denture resin [5]. Special materials are used as polymers boosters such as particles in different type; metals and metals oxide [6], $\mathrm{Al}_{2} \mathrm{O}_{3}$ [7], $\mathrm{TiO}_{2}$ [8], $\mathrm{ZrO}_{2}$ and $\mathrm{Pd}$ [4]. In addition, different type of food peels [9, 10, 11]. Were used. 
According to the fact that fiber reinforcement leads to enhance the strength of polymer matrix [12]. Jawad K. Oleiwi et al. (2017) investigated the qualities of PMMA reinforced with Siwak fibers and found that the tensile properties improved. The fibers were cut into three lengths and various concentrations were employed. The results showed that the tensile characteristics of reinforcing fiber and loading fillers improved with length. [13, 14].

Fahmi and, Ebrahim in (2017), evaluated the influence of reinforcement of heat-cure poly (methyl methacrylate) resin by polypropylene fibers with percentage of $10 \%$ by weight, on Flexural Strength, Fracture Toughness and hardness properties. The results showed that by addition of polypropylene fibers lead to increase the mechanical properties of heat- polymerized acrylic resin. The results were statistically analyzed by one-way analysis of variance (ANOVA) and Tukeys test [15].

Oleiwi et. al. (2018), evaluated the effect of Siwak and Bamboo fibers as reinforcing materials on impact and compression strength of denture base resin. The additives were in three percentages $(3,6,9 \%) \mathrm{Wt}$. by weight, and they concluded that the impact strength of PMMA acrylic resin decreasing with increasing the weight fraction of reinforcing material while the compression strength increased. Increasing the fiber length for both types resulted in increasing the impact strength and decreasing compression strength [16].

Okeke, et. al. (2018), studied the flexural and impact strength of denture base resin reinforced by Hibiscus sabdariffa fiber with different weight fraction as $(2.5,5,7.5,10 \%) \mathrm{Wt}$., the results showed that the reinforcement by Hibiscus sabdariffa improved flexural strength and strength the denture base resin and the specimens reinforced by (7.5\%) Wt., to represent the highest value. Statistically ANOVA and Bonferroni tests showed significant differences among the groups [17].

Also, different type of nanotube were utilized to improve the dental material properties such as; Carbon nanotubes (CNTs), $\mathrm{TiO}_{2}$ nanotubes, $\mathrm{ZrO}_{2}$ nanotubes and halloysite nanotubes (HNT) Copper nanotube CNTs used due to the advance properties; CNTs specially used because it considered strong, resilient, and lightweight [12, 18].

The aim of the study was to develop advance denture resin materials that are more biocompatible with better physical characteristics, from as PMMA/PP and PMMA/PAN composites. In this work, evaluate the (FTIR) behavior of the produced composite and investigate the influence of these additional fibers with various weight ratios on physical characteristics such as water absorption, thermal conductivity, and density.

\section{Materials and Methods}

\subsection{Matrix Material}

In this research, a self-curing resin (Duracryl Plus) was used that Manufactured by Spofa Dental (made in Czech Republic as is a manufacturer of non-metallic dental materials. The company belongs to the KAVOKERR group of companies, which is part of Envista Holding Corporation). This type of resin has many properties such as: low molecular weight, color stable in the long run, minimized shrinkage, softer feel, stable polymerization cycle with a perfect end result. The acrylic is long lasting and moveable for a long period of time [19]. Two types of fibers Polypropylene (PP) and Poly acrylonitrile (PAN) were used as reinforcing materials to PMMA with weight fraction of $(1.5,3.5,5.5$ and $7.5 \%) \mathrm{Wt}$.

\subsection{Composite Specimens Preparation}

The matrix polymers (PMMA) is prepared by mixing self-curing base resin in a volume ratio of 3:1 (three parts of powder(PMMA), one part of liquid(monomer)). The PMMA composite were prepared by adding (PP) or (PAN) fibers to the matrix according to the selected ratio $(1.5,3.5,5.5$ and $7.5 \%) \mathrm{Wt}$. Using a balance of scale $(0.0001)$ the total weight of PMMA and other additive were measured according to the mixture base the used mold. Essentially, the fibers were treated with $5 \%$ (weight /volume) alkaline solution $\mathrm{Na} \mathrm{OH}$ for 24 hours at room temperature. Then, the alkaline treated fibers were washed with distilled water to get rid of the additional $\mathrm{NaOH}$ solution adhering to their surface. Finally, they were dried for two days at room temperature, put on an oven at nearly $\left(65^{\circ} \mathrm{C}\right)$ to dry. 
The mixture of one type of (PP) or (PAN) fibers with (PMMA) powder first added according to the selected glass mold dimension $\left(15 \times 20 \times 0.4 \mathrm{~cm}^{3}\right)$. Later on, the monomer liquid is added (MMA) to the specified amount to each mixture secondly at room temperature, with homogeneous stirring for about two minutes until the dough stage. Third, all samples are dried at room temperature for one-day period to remove the residual pressure due to loosening of the samples from the mold cavity. All samples are placed in a drying oven at a temperature of $65^{\circ} \mathrm{C}$ for 30 minutes, then they are cut precisely using a $(\mathrm{CNC})$ machine and immersed in distilled water at $37^{\circ} \mathrm{C}$ for 48 hours (specification ADA.1999,No. 12) [20]. Finally, the samples examined according to the required characteristics.

\section{Physical and Miscibility Properties}

\subsection{Fourier Transform Infrared Spectra (FTIR) Test}

The Fourier transform infrared spectrum is utilized to extract precise information about polymer samples' chemical bonding and molecular structure. The FTIR test was carried out in accordance with international measurements (ASTM E-1252) [21], utilizing a Brukeroptier type (Fourier infrared) spectrometer (TENSOR-27). The infrared spectrum was utilized in the $\left(400-4000 \mathrm{~cm}^{-1}\right)$ range.

\subsection{Water Absorption Test}

In this test, the sample is weighed using an accurate balanced scale (0.0001), and then the samples are immersed in distilled water for 24 hours at room temperature according to specifications (ASTM D570) [22]. A dry cloth is used to dry the sample after leaving the distilled water. After that, all samples were weighed again and using equation (1) to obtain water absorption value for each case. Figure 1 shows the standard sample and the composite samples from this test [23].

$$
\text { Water absorption percentage }=\quad\left(W_{s}-W_{d}\right) / W_{d} \times 100
$$

where: Wd: Mass of the specimen before immersion (dry), Ws: Mass of the specimen after immersion for (24 hr. in distilled water).

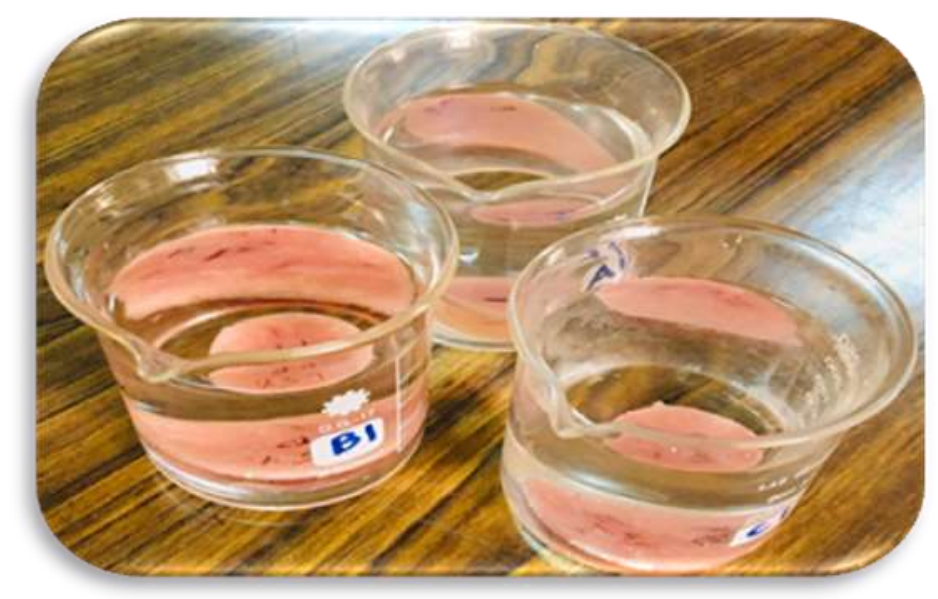

Figure 1: Water Absorption Test.

\subsection{Thermal Conductivity Test}

Thermal conductivity of composite samples represents the movement in the molecular chains of polymer in composite specimens due to thermal energy at a rate proportional to the content of the conductive materials [24]. Also, thermal conductivity is defined as heat transferred from a high to low temperature region of a material. Conductivity property is measured using thermal conduction devices as in the following equation [25].

$$
J_{E=-K} \frac{d r}{d x}
$$


where: represents the heat flux, or heat flow, per unit time per unit area (where the area is considered as that perpendicular to the flow direction), $\mathrm{J}_{\mathrm{E}}$ : power current density, $\mathrm{k}$ : Thermal conductivity property, $\mathrm{dr} / \mathrm{dx}$ : Temperature gradient through the conductive medium.

Therefore, to measure the heat flowing within the cross-sectional area of the sample per unit time a Lee disk device is used as in the following equation:

$$
k\left[\frac{T_{B}-T_{A}}{d s}\right]=e\left[T_{A}+\frac{2}{r}\left(d_{A}+\frac{1}{4 d s}\right) T_{A}+\frac{1}{2 r} d s T_{B}\right]
$$

where: I represents current value through the electrical circuit, while $\mathrm{V}$ is the voltage supplied. Then, thermal conductivity $\mathrm{K}(\mathrm{W} / \mathrm{m} . \mathrm{K})$ was measured using the following equation:

$$
I V=\pi r^{2} e\left(T_{A}+T_{B}\right)+2 \pi r e\left[d_{A} T_{A}+d_{s} \frac{1}{2}\left(T_{A}+T_{B}\right)+d_{B} T_{B}+d_{c} T_{c}\right.
$$

where $\mathrm{T}_{(\mathrm{A}, \mathrm{B}, \mathrm{C})}$ are the temperatures of $\mathrm{A}, \mathrm{B}$ and $\mathrm{C}$ brass discs, whereas $\mathrm{d}_{(\mathrm{A}, \mathrm{B}, \mathrm{C})}$ are their thickness values, respectively.

\subsection{Density Test}

The density of the samples was tested using Archimedes' rule with balances kind: PS 360/C/1device [26], by measuring composite samples weights as the standard (D-792)[27]. The density test is one of the important tests that show the lightness of the denture sample weights.

\section{Results and Discussion}

To thoroughly characterize the band of PMMA composite specimens, Fourier transforms infrared spectroscopy (FTIR) results shown in Figures 2,3, and 4 were used. The infrared spectra of neat PMMA is shown in Figure 2, with the asymmetric and symmetric peaks corresponding to the $(\mathrm{C}-\mathrm{H})$ stretching of the methyl group $\left(\mathrm{CH}_{3}\right)$ at 1339.84 and $1579.53 \mathrm{~cm}^{-1}$, respectively. Furthermore, $\mathrm{C}=\mathrm{O}$ and $\mathrm{C}-\mathrm{O}$ bands can be found in the ranges of $1500-2000 \mathrm{~cm}^{-1}$ and 1000 $1400 \mathrm{~cm}^{-1}$ [26]. As a result, the bands at $2919.52 \mathrm{~cm}^{-1}$ and the medium strength of $\mathrm{C}=\mathrm{O}$ stretching at $1716.66 \mathrm{~cm}^{-1}$ are associated with $(\mathrm{C}-\mathrm{H})$ symmetric and asymmetric stretching modes, respectively. The torsion of the methylene group $\mathrm{CH}_{2}$ is ascribed to the $1339.84 \mathrm{~cm}^{-1}$ band, and the peak at $1253.13 \mathrm{~cm}^{-1}$ relates to vibration of the ester group $(\mathrm{C}-\mathrm{O})$, while the $(\mathrm{C}-\mathrm{C})$ stretching bands are at 1040.63 and $976.17 \mathrm{~cm}^{-1}[28,29]$. The medium strength of $\mathrm{C}=\mathrm{O}$ bending is ascribed between 1579.53 and $1599.53 \mathrm{~cm}^{-1}$ in this spectrum. Figures $3 \& 4$ show the infrared spectrum of neat PMMA and PMMA composite reinforced with various weight fractions of (PP and PAN) fibers of 1.5, 3.5, 5.5, and 7.5 \% Wt. All features of PMMA composites vibration bands assigned in the infrared spectrum (FTIR) for all composites specimens developed for this study are shown in these figures.

The FTIR results for all specimens are shown in these figures, and it can be seen that no new peaks formed following the fiber strengthening. Furthermore, there was no movement in any of these summits. This tendency leads to the formation of physical bonds between composite constituents, which is a good indicator of how the miscibility state of composite constituents is changing. Approve the absence of any leftover monomer or other secondary impact as a result of the product that could induce cytotoxicity, allergic reactions, or inflammation in humans.

The infrared spectrum (FTIR) for PMMA specimens reinforced with PP fiber at various weight fractions (1.5, 3.5, 5.5, and $7.5 \%) \mathrm{Wt}$ is shown in Figure 3. It shows that the peak intensity of neat PMMA was minimized, and that the peak intensity of all PMMA composite characteristics increased as the weight fraction of PP fiber increased. It was able to obtain the maximum value of clean PMMA. In all percentages of addition, the peak intensity of PMMA composite declines to less than that of pure PMMA. The infrared spectrum (FTIR) for PMMA composite specimens enhanced by PAN fiber at different weight fractions $(1.5,3.5,5.5$, and $7.5 \%) \mathrm{Wt}$ is shown in Figure 4. Furthermore, when the weight fraction of PAN fibers increases, the peaks intensity of PMMA composite becomes higher than that of neat PMMA, until it reaches its maximum value at $5.5 \% \mathrm{Wt}$. 


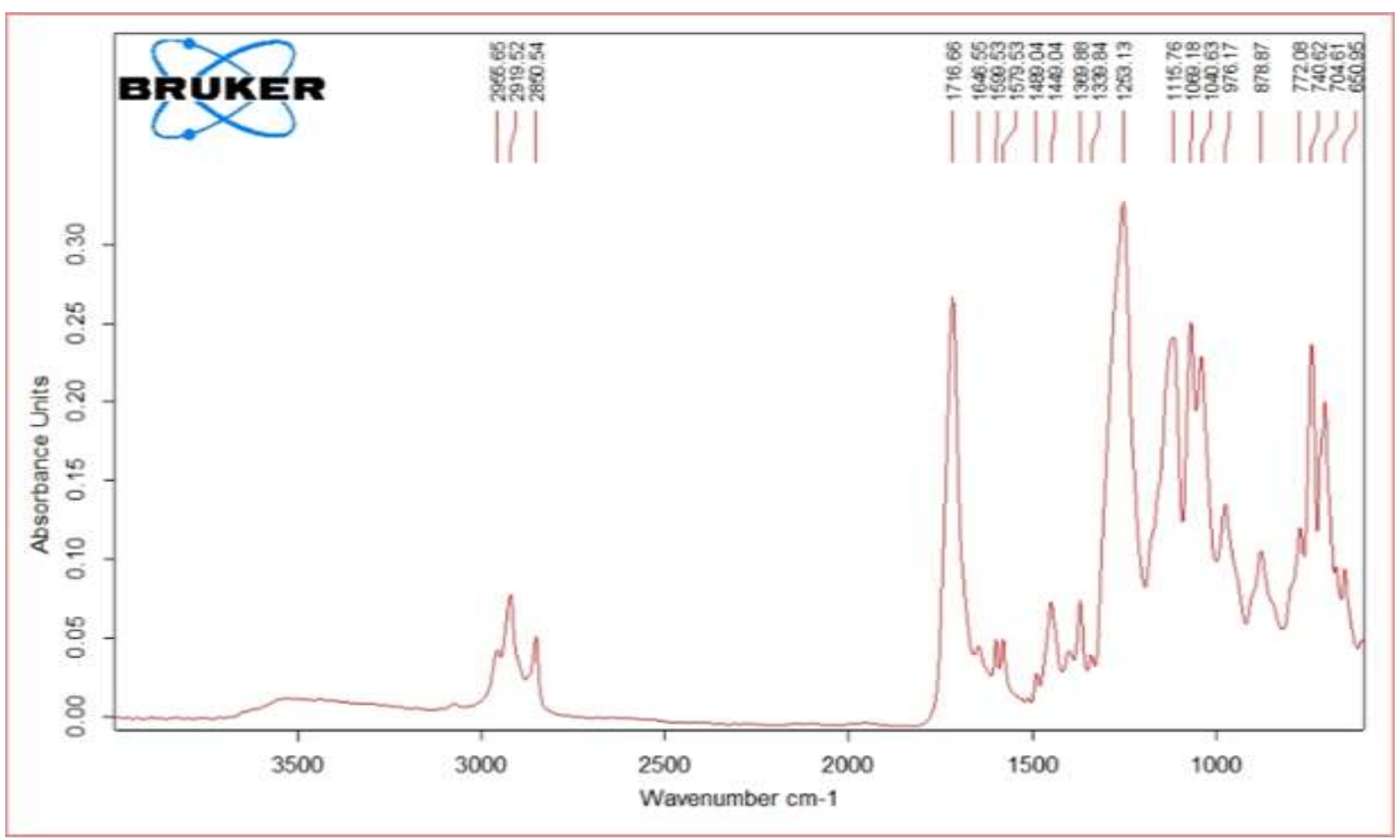

Figure 2: The Infrared Spectrum that obtained for the self - Cure PMMA Specimens.

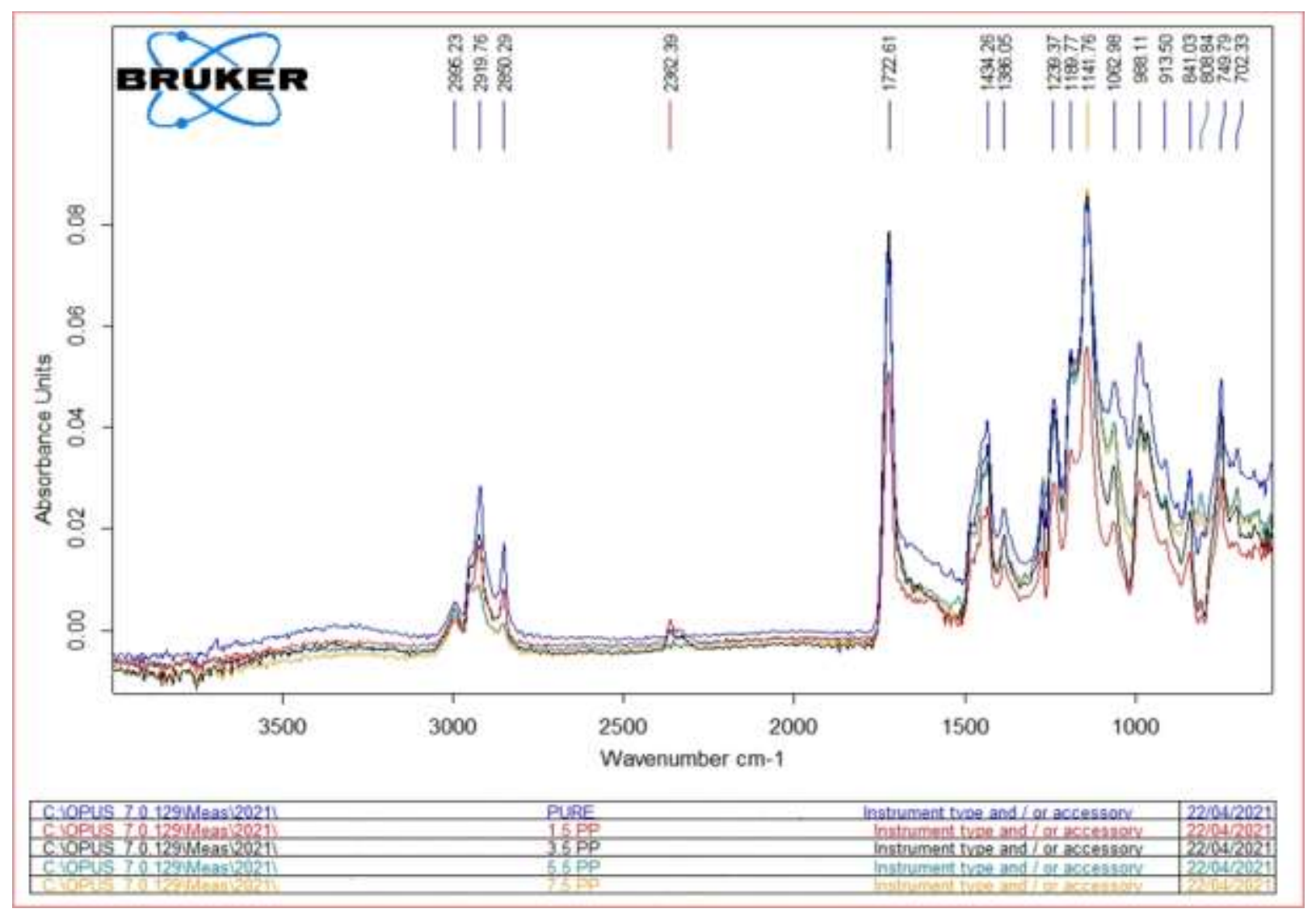

Figure 3: Represents FTIR Spectrum of PMMA Composite Reinforced with PP fiber. 


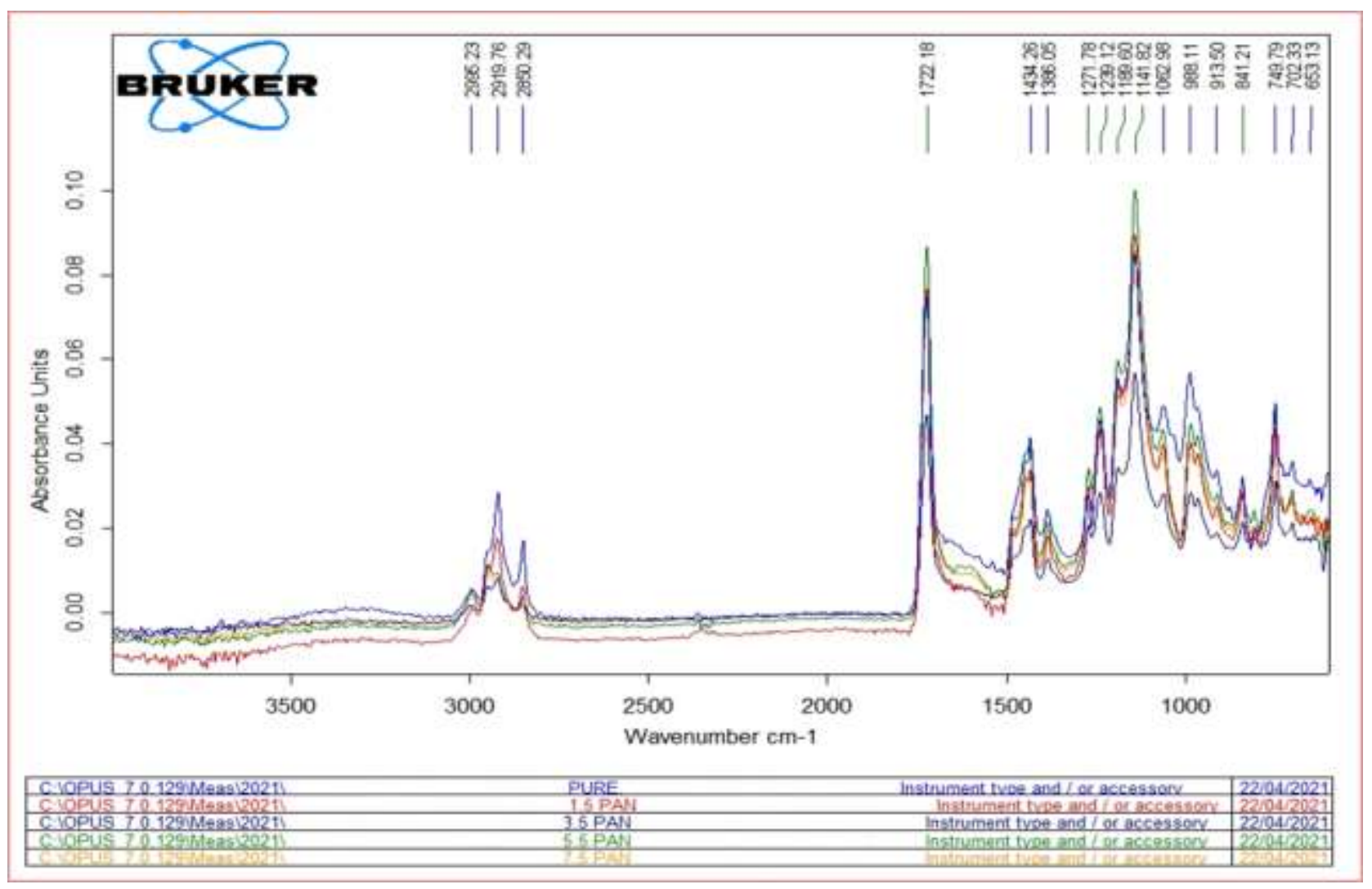

Figure 4: Represents FTIR Spectrum of PMMA Composite Reinforced with PAN fiber.

Water absorption test show in figure 5 illustrate the relationship between the water absorption and the reinforcement ratio of the PMMA/PP and PMMA/PAN composite. It is evident from the shape that the water absorption gradually increases during the increase in the used fibers ratio compared to the neat polymer sample (PMMA). Furthermore, there was no change in all samples dimensions. Yet, a noticeable increase in composite total weight measured according to both (PP and PAN) weight fraction increase due to the fiber's encounter with moisture, and it may also be due to the fibers high level of moisture absorption compared to the polymer matrix resulting from Polar hydroxide groups in fibers [30]. Hence, it could register the maximum increase in this property at the highest reinforcement percentage which was $7.5 \% \mathrm{Wt}$., for both types of fibers. Water absorption at the maximum fiber addition gave $0.6 \%$ compared to the neat sample which was $(0.3 \%)$. In general, PMMA/PP composite possess a higher water absorption rate compared to PMMA/PP, since the composite sample with PP fibers have lower density than composite sample with PAN fibers. Interestingly, moisture absorption increase with density decrease [31, 32]. 


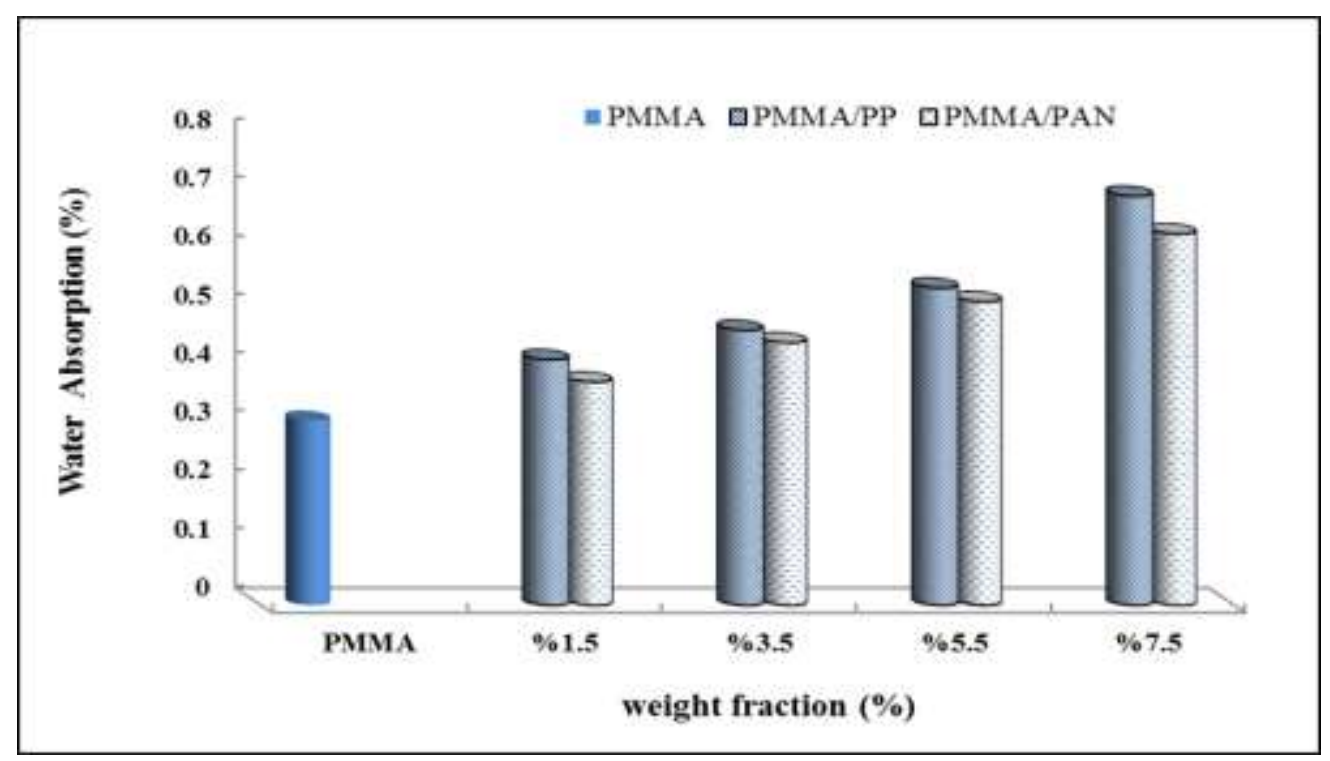

Figure 5: Water absorption value as a function of wt.\% of PP and PAN fibers.

Thermal conductivity test shows in figure 6 shows a comparison between the thermal conductivity values of all samples as a function to the fiber ratios. It is evident from the figure that the thermal conductivity value decreases with the increase in the fiber reinforcement ratio compared to the neat sample. This is due to the fact that the used fillers have lower thermal conductivity compared with the PMMA matrix, which led to decrease the composite property. PP fiber thermal conductivity were found equal to $(0.12 \mathrm{~W} / \mathrm{m} . \mathrm{K})$, while PAN fibers value found as $(0.23 \mathrm{~W} / \mathrm{m} . \mathrm{K})[33$, 34]. In addition, it could be attributed to the fact of the spaces and voids between the filaments that exist in both types of fibers that lead to difficulties in heat transfer procedure through the composite materials [35]. Also, this behavior could be due to the poor interfacial adhesion within the polymer matrix upon the increase of the additive percentage [9]. Thermal conductivity was decreased at the highest fibers ratio $7.5 \% \mathrm{Wt}$., for the PAN. $(0.24 \mathrm{~W} / \mathrm{m} . \mathrm{K})$ and PP fibers that present slightly higher than that $(0.33 \mathrm{~W} / \mathrm{m} . \mathrm{K})$ compared to the neat sample $(0.46 \mathrm{~W} / \mathrm{m} . \mathrm{K})$.

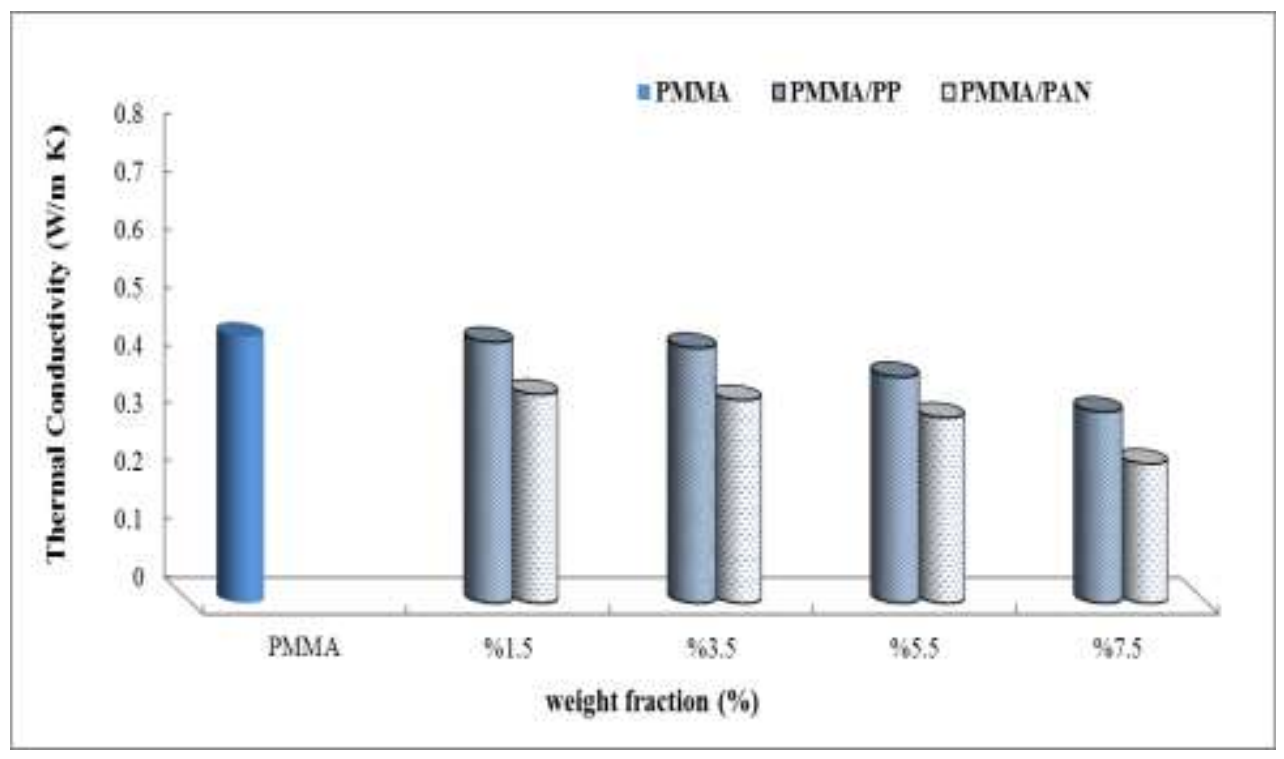

Figure 6: Thermal conductivity value as a function of wt.\% of PP and PAN fibers. 
The density test shows in figure 7 for the relationship between density and weight fraction of added fibers. The density value of both PMMA/PP and PMMA/PAN composite samples was tested and compared with the neat PMMA polymer. It was noticed that a reduction in the density values within the increase of both the reinforcement fibers percentage. The density of neat PMMA was equal to $\left(1.16 \mathrm{~g} / \mathrm{cm}^{3}\right)$, while the density value for both composite decreased as the additive increased, PMMA/PP had the lowest value at the same circumstances until at the addition (7.5) \%Wt., weight fraction both composite presented the $\left(1.09 \mathrm{~g} / \mathrm{cm}^{3}\right)$ for PMMA/PP and $\left(1.1 \mathrm{~g} / \mathrm{cm}^{3}\right)$ PMMA/PAN. The reason behind that, both fibers densities were much lower compared to determined PMMA. Through literature, it was found that PP fiber and PAN fiber density were about $\left(0.9 \mathrm{~g} / \mathrm{cm}^{3}\right)$ and $\left(1.8 \mathrm{~g} / \mathrm{cm}^{3}\right)$ respectively $[33,36]$.

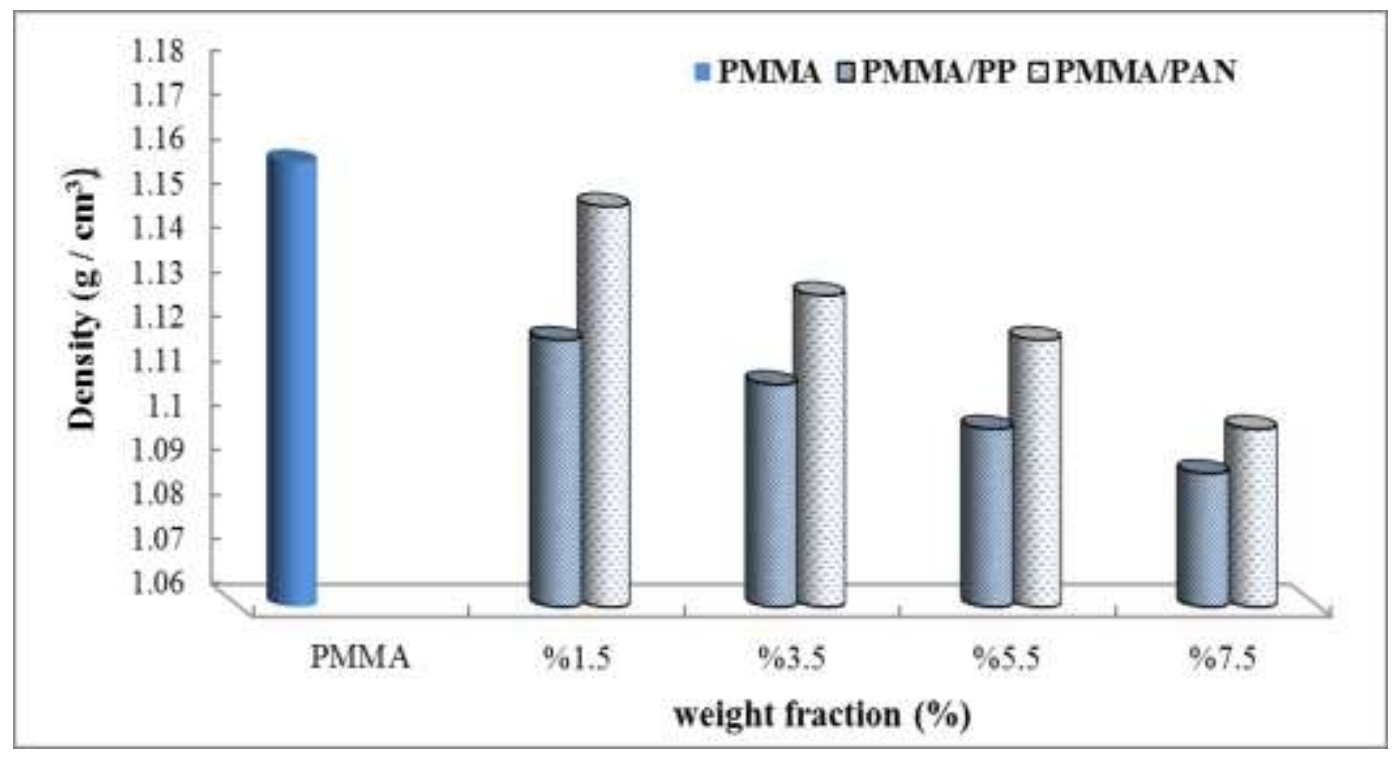

Figure 7: Density value as a function of wt.\% of PP and PAN fibers.

\section{CONCLUSIONS}

The reinforcement effect on neat PMMA which specifically used to fabricate denture base with two type of synthetic fibers (PP and PAN) at different weight fraction were studies, the following results conclude:

Water absorption ability increased significantly with the increase in the both fibers ratio, the highest value determined was at $(7.5 \%) \mathrm{Wt}$., for both fibers determined over $(0.6 \%)$ compared to the neat sample $(0.3 \%)$. On the other hand, a gradual decline in the thermal conductivity property was obtained through the increase of fiber ratio, at the highest ratio for PAN fiber $(0.24 \mathrm{~W} / \mathrm{m}$. K) compared to PP fiber $(0.33 \mathrm{~W} / \mathrm{m} . \mathrm{K})$, while the neat PMMA polymer determined $(0.46 \mathrm{~W} / \mathrm{m} . \mathrm{K})$. Also, it observed that a noticeable decrease in the density while the fibers ratios were increased with all composite samples. Density for PMMA/PP $\left(1.09 \mathrm{~g} / \mathrm{cm}^{3}\right)$ and for PMMA/PAN $\left(1.1 \mathrm{~g} / \mathrm{cm}^{3}\right)$ at the ratio $7.5 \% \mathrm{Wt}$., compared to the neat sample $\left(1.16 \mathrm{~g} / \mathrm{cm}^{3}\right)$. Finally, FTIR results had been showed that, there were no new peaks, appeared after the reinforcing by both type of fibers PP and PAN. Also, this behavior refers to the good physical bond between the matrix and the reinforcing material, and there was no new material formed

\section{Acknowledgement}

The authors express their sincere appreciation to the Applied Science Department and Material Engineering Department, University of Technology, Baghdad, Iraq.

\section{Conflict of Interest}

There are no conflicts of interest regarding the publication of this manuscript. 


\section{References}

[1] Q. A. Hamad, "Study the Effect of Nano Ceramic Particles on Some Physical Properties of Acrylic Resins," Engineering and Technology Journal, vol. 35, no. 2 Part A, 2017.

[2] S. Chen, W. Liang, and P. Yen, "Reinforcement of acrylic denture base resin by incorporation of various fibers," Journal of Biomedical Materials Research: An Official Journal of The Society for Biomaterials, The Japanese Society for Biomaterials, and The Australian Society for Biomaterials and the Korean Society for Biomaterials, vol. 58, no. 2, pp. 203-208, 2001.

[3] R. K. Alla, S. Sajjan, V. R. Alluri, K. Ginjupalli, and N. Upadhya, "Influence of fiber reinforcement on the properties of denture base resins," 2013.

[4] M. M. Gad, S. M. Fouda, F. A. Al-Harbi, R. Näpänkangas, and A. Raustia, "PMMA denture base material enhancement: a review of fiber, filler, and nanofiller addition," International journal of nanomedicine, vol. 12, p. 3801, 2017.

[5] A. M. El-Sheikh, "Causes of denture fracture: A survey," Saudi Dent J, vol. 18, pp. 149-154, 2006.

[6] Z. Han, B. Zhu, R. Chen, Z. Huang, C. Zhu, and X. Zhang, "Effect of silver-supported materials on the mechanical and antibacterial properties of reinforced acrylic resin composites," Materials \& Design (19802015), vol. 65, pp. 1245-1252, 2015.

[7] Z. N. Rasheed and S.M. Hussain, "Impact strength behaviour of PMMA denture base through addition of different nanoparticles after immersion in some nutrition liquids," Iraqi Journal of Physics (IJP), vol. 17, no. 41, pp. 40-50, 2019.

[8] S. A. Alwan and S. S. Alameer, "The effect of the addition of silanized Nano titania fillers on some physical and mechanical properties of heat cured acrylic denture base materials," Journal of baghdad college of dentistry, vol. 325, no. 2218, pp. 1-12, 2015.

[9] Z. N. R. Alraziqi and H. S. Mansoor, "Experimental Investigation for Some Properties of PMMA Denture Base Strengthened by Different Nanoadditives," Iraqi Journal of Science, pp. 2913-2925, 2020.

[10] J. K. Oleiwi, Q. A. Hamad, and N. N. Kahdim, "Study Compression, Hardness and Density properties of PMMA Reinforced by Natural Powder Used in Denture Base applications," Engineering and Technology Journal, vol. 37, no. 12A, pp. 522-527, 2019.

[11] S. M. Hussein and Z. N. Rasheed, "Nano-reinforcement effect on PMMA denture base mechanical properties after immersing in different solution," Iraqi journal of science, pp. 57-68, 2019.

[12] N. Prajwala, C. R. Kumar, M. Sujesh, D. C. Rao, and L. Pavani, "Denture base reinforcing materials-A review," IP Annals of Prosthodontics and Restorative Dentistry, vol. 6, no. 2, pp. 52-59, 2020.

[13] J. K. Oleiwi, S. I. Salih, and H. S. Fadhil, "Effect of siwak and bamboo fibers on tensile properties of selfcure acrylic resin used for denture applications," J Material Sci Eng, vol. 6, no. 370, pp. 22-2169, 2017.

[14] S. I. Salih, J. K. Oleiwi, and H. S. Fadhil, "Preparation and investigation of some properties of acrylic resin reinforced with siwak fiber used for denture base applications," Kurdistan Journal of Applied Research, vol. 2, no. 3, pp. 309-314, 2017.

[15] M. Fahmi and M. Ebrahim, "Effect of polypropylene fiber addition on the flexural strength, fracture toughness, and hardness of heat-polymerized acrylic resin," EC Dent Sci, vol. 8, no. 3, pp. 101-109, 2017.

[16] J. K. Oleiwi, S. I. Salih, and H. S. Fadhil, "Study Compression and Impact Properties of PMMA Reinforced by Natural Fibers Used in Denture," Engineering and Technology Journal, vol. 36, no. 6 Part A, pp. 652- 
$655,2018$.

[17] K. N. Okeke, A. Vahed, and S. Singh, "Improving the strength properties of denture base acrylic resins using hibiscus sabdariffa natural fiber," Journal of International Dental and Medical Research, vol. 11, no. 1, pp. 248-254, 2018.

[18] R. Wang, J. Tao, B. Yu, and L. Dai, "Characterization of multiwalled carbon nanotube-polymethyl methacrylate composite resins as denture base materials," The Journal of prosthetic dentistry, vol. 111, no. 4, pp. 318-326, 2014.

[19] W. J. O’Brien, "Dental materials and their selection," 2002.

[20] C. on D. M. and Devices, "Revised American dental association specification no. 12 for denture base polymers," The Journal of the American Dental Association, vol. 90, no. 2, pp. 451-458, 1975.

[21] A. E1252, "Standard Practice for General Techniques for Obtaining Infrared Spectra for Qualitative Analysis," Annual Book of Standards, 2013.

[22] A. C. D.-20 on P. S. D. 50 on P. Properties, Standard Test Method for Water Absorption of Plastics. American Society for Testing and Materials, 1995.

[23] M. A. M. M. Idrus, S. Hamdan, M. R. Rahman, and M. S. Islam, "Liquefied tropical wood/polypropylene composites: Preparation and physico-mechanical properties," Materials Physics and Mechanics, vol. 11, pp. 126-136, 2011.

[24] D. R. Askeland, P. P. Phulé, W. J. Wright, and D. K. Bhattacharya, "The science and engineering of materials," 2003.

[25] R. A. Nassif, "Effect of chemical treatment on the some electrical and thermal properties for unsaturated polyester composites using banana fibers," matrix, vol. 1, p. 7, 2010.

[26] C. E. Carraher Jr, Introduction to polymer chemistry. CRC press, 2012.

[27] ASTM D 792-20, "Standard Test Methods for Density and Specific Gravity ( Relative Density ) of Plastics," Annual Book of ASTM Standards, vol. 08.01, pp. 1-6, 2020.

[28] F. Chen, Z.-C. Wang, and C.-J. Lin, "Preparation and characterization of nano-sized hydroxyapatite particles and hydroxyapatite/chitosan nano-composite for use in biomedical materials," Materials letters, vol. 57, no. 4, pp. 858-861, 2002.

[29] D. E. Baciu, J. Simitzis, and D. G. Opowlos, "Synthesis and characterization of acrylic bone cement reinforced with zirconia bio ceramic," Digest Journal of Nanomaterials and Bio structures, vol. 7, no. 40, pp. 1779-1786, 2012.

[30] G. Das and S. Biswas, "Physical, mechanical and water absorption behaviour of coir fiber reinforced epoxy composites filled with $\mathrm{A} 12 \mathrm{O} 3$ particulates," in IOP conference series: materials science and engineering, 2016, vol. 115, no. 1, p. 12012.

[31] J. K. Oleiwi, S. I. Salih, and H. S. Fadhil, "Water Absorption and Thermal properties of PMMA Reinforced by Natural Fibers for Denture Applications," International Journal of Mechanical and Production Engineering Research and Development, vol. 8, no. 3, pp. 1105-1116, 2018.

[32] M. Sumaila, I. Amber, and M. Bawa, "Effect of fiber length on the physical and mechanical properties of ramdom oriented, nonwoven short banana (musabalbisiana) fiber/epoxy composite," Cellulose, vol. 62, p. 64, 2013. 
[33] O. Alidoust, I. Sadrinejad, and M. A. Ahmadi, “A study on cement-based composite containing polypropylene fibers and finely ground glass exposed to elevated temperatures," J World Acad Sci, vol. 34, 2007.

[34] W. S. Khan, R. Asmatulu, I. Ahmed, and T. S. Ravigururajan, "Thermal conductivities of electrospun PAN and PVP nanocomposite fibers incorporated with MWCNTs and NiZn ferrite nanoparticles," International Journal of Thermal Sciences, vol. 71, pp. 74-79, 2013.

[35] S. Fu and Y. Mai, "Thermal conductivity of misaligned short-fiber-reinforced polymer composites," Journal of applied polymer science, vol. 88, no. 6, pp. 1497-1505, 2003.

[36] K. Naito, J.-M. Yang, Y. Xu, and Y. Kagawa, "Enhancing the thermal conductivity of polyacrylonitrile-and pitch-based carbon fibers by grafting carbon nanotubes on them," Carbon, vol. 48, no. 6, pp. 1849-1857, 2010 . 